\title{
Cytogenetic evaluation of tannery workers in the city of Teresina, northeastern Brazil
}

\author{
F.P.M. do Amaral', E.J.L.S. do Amaral', M.P.M. do Amaral' ${ }^{1}$, L.S. Lopes ${ }^{1}$, \\ S.I. Lemos ${ }^{1}$, R.V.G. Santos ${ }^{1}$, T.V.S. Chaves ${ }^{2}$, M.E.A. de Moraes ${ }^{3}$ and \\ M.O.A. de Moraes Filho ${ }^{3}$ \\ 'Laboratório de Biologia Molecular e Estudo de Injúrias Biológicas, \\ Faculdade de Ciências Médicas, Universidade Estadual do Piauí, Teresina, PI, Brasil \\ ${ }^{2}$ Centro de Referência em Saúde do Trabalhador, Secretaria do Estado do Piauí, \\ Teresina, PI, Brasil \\ ${ }^{3}$ Laboratório de Oncologia Experimental e Unidade de Farmacologia Clínica, \\ Universidade Federal do Ceará, Fortaleza, CE, Brasil \\ Corresponding author: F.P.M. do Amaral \\ E-mail: fabricio34amaral@gmail.com
}

Genet. Mol. Res. 14 (4): 18799-18807 (2015)

Received June 18, 2015

Accepted September 2, 2015

Published December 28, 2015

DOI http://dx.doi.org/10.4238/2015.December.28.29

\begin{abstract}
Assessments of chromosomal integrity and structure enable the prevention of diseases associated with the work environment, with the frequencies of chromosomal aberrations and micronuclei often being used as markers in biomonitoring. Owing to their routine manipulation of potentially toxic chemicals, tannery workers as a group merit a more thorough evaluation and discussion. This study investigated chromosomal damage in 30 workers from a tannery in the city of Teresina, the state capital of Piauí, northeast Brazil, and a control group consisting of 30 employees from a nearby accounting firm. The frequencies of chromosomal aberrations (CAs) and binucleated cell micronuclei (MN) were assessed as a measure of damage. Means were compared using the Student $t$-test and ANOVA-Dunnett test. Our results indicated a higher number of CAs in exposed individuals compared to the control group, including dicentric $(P<0.0001)$ and tricentric chromosomes $(P<0.001)$, and those in ring
\end{abstract}


$(P<0.0001)$ and acentric ring forms $(P<0.001)$. Assessment of $M N$ frequency demonstrated a similar trend (exposed vs control, $\mathrm{P}<0.0001$ ). It was concluded that the tannery workers in this study exhibited a higher incidence of genetic damage than comparable unexposed individuals. However, further research on this subject is needed, particularly in regard to potentially clastogenic agents used in the tanning process.

Key words: Tannery workers; Chromosomal aberrations; Micronuclei

\section{INTRODUCTION}

Tannery workers are routinely exposed to a large number of chemical agents. For this reason, various studies have been carried out to evaluate their health. Asthma (Shahzad et al., 2006), stomach disorders (Rastogi et al., 2008), and increased cancer incidence (Veyalkin and Gerein, 2006) have all been identified in epidemiological investigations, and have been associated with exposure to the metal chromium (Rastogi et al., 2008). In addition, genetic studies have obtained evidence that an environmental agent is responsible for altering cellular integrity in such individuals, and possibly contributing to the onset of these diseases. Research such as that conducted by Hamamy et al. (1987), Hilali et al. (2008), Monteiro Neto et al. (2010), and Balachandar et al. (2010) has addressed the issue of chromosomal aberrations (CAs) in tannery workers. Although initial results obtained by Hamamy et al. (1987) indicated an absence of genetic alterations in this group, later studies produced contradictory results, demonstrating that further investigation was required. In Brazil, Monteiro Neto et al. (2010) evaluated 10 employees of a tannery in the city of Franca in São Paulo State, southeast Brazil. Their results revealed a higher frequency of CAs in individuals exposed to tannery chemicals. This finding corroborates those found in the literature following the Hamamy et al. (1987) investigation. In order to contribute to the body of knowledge relating to this issue, a cytogenetic evaluation of tannery workers exposed to chemical hazards in the city of Teresina, northeast Brazil, was carried out.

\section{MATERIAL AND METHODS}

\section{Subjects}

Our study included 30 tannery employees and 30 unexposed individuals employed at a local accounting office, for a total $N=60$. The former participated directly in the tanning process, working on the separation area, wet blue, semi-finished, and production stages, with no specific role. Questionnaires were used to collect personal (age, working hours, health details, etc.) and lifestyle data (eating habits, alcohol and tobacco consumption, etc.) from both groups.

\section{Study site}

Teresina, state capital of Piauí (Figure 1), is located inland in a region known as the midnorth of Brazil. According to the Brazilian Institute of Geography and Statistics (IBGE, 2014), the estimated population is 840,600 , covering $1,391,981 \mathrm{~km}^{2}$, and has a human development index, 0.751 . The city is a reference for its high standard of healthcare. Teresina has a functioning tannery, 
however, according to data from the Tanning Industry Center of Brazil (CIC, 2013), Piauí was ranked 12th in Brazil for leather exports between the years 2011 and 2013.

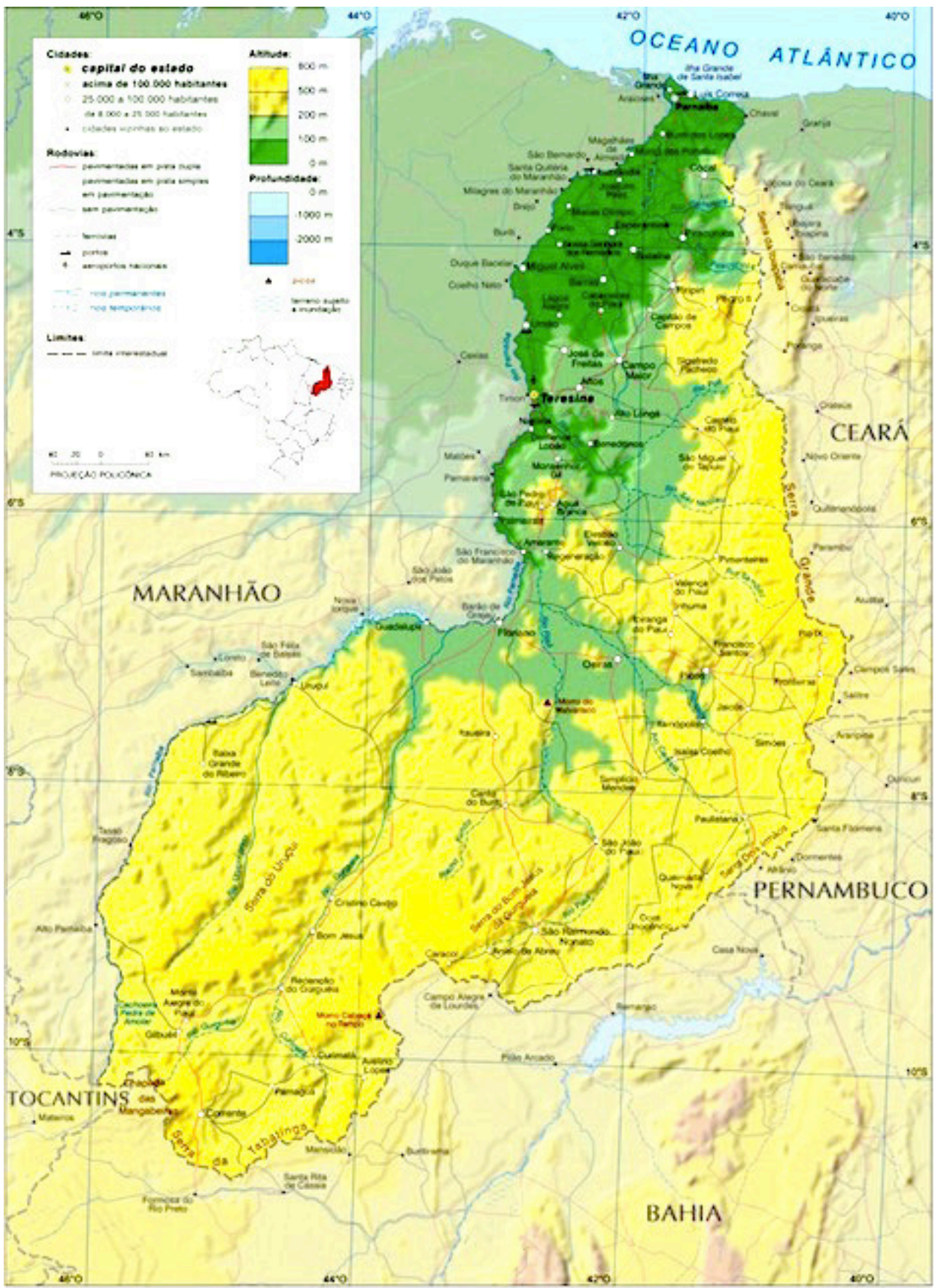

Figure 1. Piauí State with its capital, Teresina, highlighted. 
The tannery is located at 380 Avenue Poty Velho, on the outskirts of the capital in the neighborhood of Santa Maria da Codipi. At the time of study, it routinely processed 1000 pieces of leather per month. Working hours were Monday to Saturday, from 07:00 to 18:00. Raw material in the form of animal hide came from the states of Ceará, Maranhão, and Piauí. There were a total 65 employees, 30 of whom were responsible for the management of the tanning process.

\section{Sample collection and lymphocyte culture}

Following completion of the questionnaire, $5 \mathrm{~mL}$ peripheral blood was collected from each study participant using sterile, heparinized syringes. Blood samples were then sent to the Laboratory of Molecular Biology and Biological Injuries Studies (LABMINBIO), Faculty of Medical Sciences, State University of Piauí (FACIME/UESPI), and the Cytogenetics Laboratory of the Federal University of Piauí (UFPI).

The lymphocyte culture protocol for the analysis of CAs and micronuclei (MN) followed the classical criteria of Moorhead et al. (1960) and Preston et al. (1987), with some modifications. Eighteen drops of leukocyte suspension were added to $5 \mathrm{~mL}$ RPMI 1640 medium supplemented with $15 \%$ fetal bovine serum, $1 \%$ penicillin and streptomycin mixture, $1 \%$ L-glutamine, and $1 \%$ phytohemagglutinin (to stimulate cell division). The tests were carried out in triplicate, i.e. three flasks each were used for CA and MN assessments. The cultures were kept in a $\mathrm{CO}_{2}$ incubator (IG 150; Jouan, Waltham, MA, USA) at $37^{\circ} \mathrm{C}$ for $72 \mathrm{~h}$.

\section{Study of CAs}

Two hours before the incubation was complete, $16 \mu \mathrm{g} / \mathrm{mL}$ colchicine (Sigma, St. Louis, MO, USA) was added to the flasks used for CA assessment. After $72 \mathrm{~h}$, the lymphocyte culture was transferred to sterile 10-mL Falcon tubes (Thermo Fisher Scientific, Waltham, MA, USA) and centrifuged at $1000 \mathrm{rpm}$ for $5 \mathrm{~min}$, following which, hypotonic solution consisting $0.075 \mathrm{M} \mathrm{KCl}$ was added. Slides were fixed in a 3:1 methanol:acetic acid (v/v) mixture and stained with $5 \%$ Giemsa solution, pH 6.8, before being viewed under an optical microscope (CX41; Olympus, Tokyo, Japan), using oil immersion and 100x magnification. One hundred metaphases per slide per individual were analyzed. Dicentric and tricentric chromosomes, those in ring and acentric ring forms, chromosomal fragments, and terminal and interstitial deletions were counted. Our analysis was based on Savage's classification (1976). The metaphase index (MI) was calculated by multiplying the number of metaphases by 100 and dividing this figure by 300 . Similarly, CA frequency was calculated by multiplying the number of CAs by 100 and dividing the resulting value by 300 .

\section{Cytokinesis-block MN test}

The evaluation of MN in binucleated cells followed the protocol established by Pastor et al. (2003) and Fenech (2007), with some adjustments. Approximately $6 \mu \mathrm{g} / \mathrm{mL}$ cytochalasin B in dimethyl sulfoxide (Sigma) was added to cultures after $44 \mathrm{~h}$ incubation. Seventy-two hours later, cells were centrifuged at $800 \mathrm{rpm}$ for $8 \mathrm{~min}$, then rinsed in RPMI 1640 medium, and treated with $4^{\circ} \mathrm{C} 0.075 \mathrm{M} \mathrm{KCl}$ hypotonic solution for $3 \mathrm{~min}$. This procedure was repeated three times. The cells were then centrifuged again and fixed in a 3:1 methanol:acetic acid (v/v) solution. This stage was repeated twice, taking care to manipulate the samples gently. Finally, cells were resuspended and 
placed on clean plates, before being incubated with $10 \%$ Giemsa stain in phosphate buffer, $\mathrm{pH}$ 6.8 , for $10 \mathrm{~min}$. Analysis was carried out under the abovementioned light microscope using 100x magnification. Approximately 1000 binucleated cells with preserved cytoplasms were evaluated for MN frequency according to the criteria of Fenech (2007).

\section{Statistical analysis}

Statistical analysis was carried out using SPSS version 17.0 (SPSS Inc., Chicago, IL, USA) and GraphPad Prism version 4.0 (GraphPad Software, La Jolla, CA, USA). Simple frequency analysis and comparison of means using the Student $t$-test, and association analysis using the chi-square test, were carried out. For assessment of CAs, we used ANOVAs followed by Dunnett's tests. $P$ values less than 0.05 were considered statistically significant.

\section{Ethical issues}

The current research protocol was approved by the Ethics Committee of the Federal University of Ceará (UFC/CE; number 195/08). Informed consent statements were signed by all participants and confidentiality was ensured.

\section{RESULTS}

Characteristics of the study population are shown in Table 1. The groups were paired and no statistically significant differences regarding mean age and working hours were observed. Table 2 shows the MI values and frequencies of the main CA types found in lymphocytes of exposed and unexposed individuals. The MI did not significantly differ between the two groups, however, the total number and frequency of CAs was significantly higher in tannery workers. The principal types of CA found in exposed individuals were dicentric and tricentric chromosomes, and those in ring and acentric ring forms. Terminal deletions and chromosomal fragments were not frequently observed. Table 3 compares MN number and frequency between exposed and unexposed workers, demonstrating that both of these measures were significantly higher in tannery employees.

No significant differences regarding $\mathrm{CAs}$ and $\mathrm{MN}$ were found between smokers and nonsmokers or between alcohol consumers and non-consumers within the tannery worker group (data not shown).

Table 1. Characteristics of exposed and unexposed groups obtained from personal health questionnaires.

\begin{tabular}{|c|c|c|c|c|c|c|}
\hline & \multicolumn{3}{|c|}{ Exposed } & \multicolumn{3}{|c|}{ Not exposed } \\
\hline & Total & Smokers & Non-smokers & Total & Smokers & Non-smokers \\
\hline $\mathrm{N}$ & 30 & $11(36.66 \%)$ & 19 (63.33\%) & 30 & $4(13.33 \%)$ & $26(86.66 \%)$ \\
\hline Alcohol drinkers & $26(86.66 \%)$ & $11 / 26$ & $15 / 26$ & $28(93.33 \%)$ & $4 / 28$ & $24 / 28$ \\
\hline Age $^{*}$ & $34.42 \pm 9.61(18-65)$ & $40.3 \pm 10.72(32-65)$ & $32.13 \pm 8.81(18-37)$ & $32.40 \pm 8.10(20-51)$ & $46.00 \pm 6.31(43-51)$ & $30.52 \pm 10.11(20-38)$ \\
\hline Hours worked/week* & $44.51 \pm 0.96(44-50)$ & $44.51 \pm 0.96(44-50)$ & $44.51 \pm 0.96(44-50)$ & $44.19 \pm 0.93(44-50)$ & $44.19 \pm 0.93(44-50)$ & $44.19 \pm 0.93(44-50)$ \\
\hline Exposure time & $8.21 \pm 5.75$ & $9.3 \pm 7.32$ & $5.32 \pm 3.45$ & 0 & 0 & 0 \\
\hline
\end{tabular}

*Data are reported as mean values with standard deviations and ranges. A P value greater than 0.05 indicated no significant differences between the tannery workers and the control group in terms of weekly working hours and age, based on the chi-square test. 
F.P.M. do Amaral et al.

18804

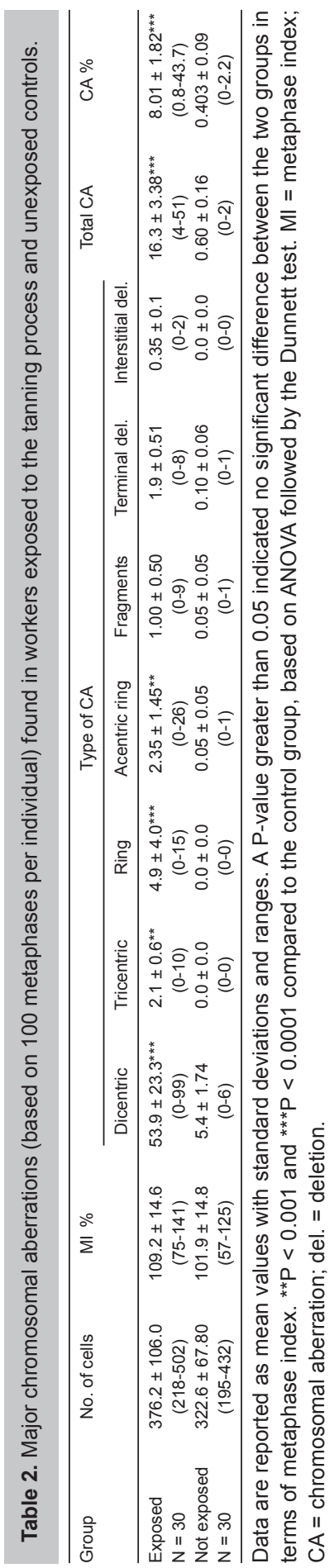


Table 3. Number and frequency of micronuclei in binucleated lymphocytes in exposed and control groups.

\begin{tabular}{lcr}
\hline Group & No. of MN per 1000 binucleated cells & MN frequency \\
\hline Exposed $\mathrm{N}=30$ & $17.73 \pm 9.44^{*}(0-33)$ & $0.886 \pm 0.472^{*}(0-1.65)$ \\
Not exposed $\mathrm{N}=30$ & $10.90 \pm 7.092(2-30)^{\mathrm{b}}$ & $0.306 \pm 0.202(0-0.75)$ \\
\hline
\end{tabular}

Data are reported as mean values with standard deviations and ranges. ${ }^{*} \mathrm{P}<0.05$ for incidence of micronuclei in binucleated cells compared to that of the control group, based on the Student $t$-test. MN $=$ micronuclei.

\section{DISCUSSION}

The relationship between CAs and neoplasia has been investigated since the 1960s (Bonassi et al., 2004). Cohort studies monitoring CAs in individuals over time and attempting to correlate them with oncogenesis have shown that environmental factors, such as occupational exposure, are not decisive in causing malignancies (Bonassi et al., 2004; Norppa et al., 2006). However, studies of tannery workers in various countries have found significant differences between the number of CAs in exposed individuals compared to unexposed controls (Hilali et al., 2008; Monteiro Neto et al., 2010). Other reports of alterations to genetic material, such as an increase in DNA lesions, have confirmed these results (Balachandar et al., 2010; Ambreen et al., 2014). In our study, dicentric, tricentric, and ring-shaped chromosomes were the most frequently observed aberrations (Figure 2). Ring chromosomes have not been detected in previous studies of tannery workers. These abnormal structures are found in many types of human neoplasia, and according to Bonassi et al. (2008), the appearance of dicentric and ring chromosomes is associated with an increased risk of carcinogenesis (with relative risks of 2.22 and 1.32 for ring and dicentric forms, respectively).

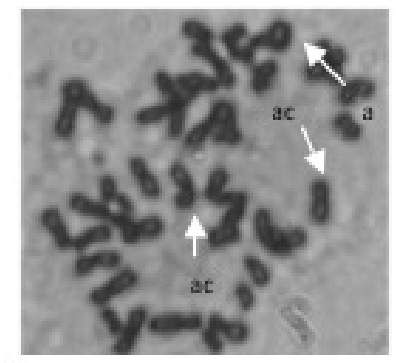

A)

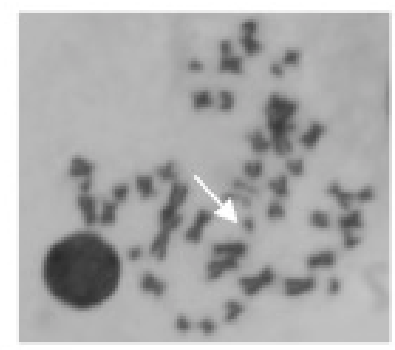

C)

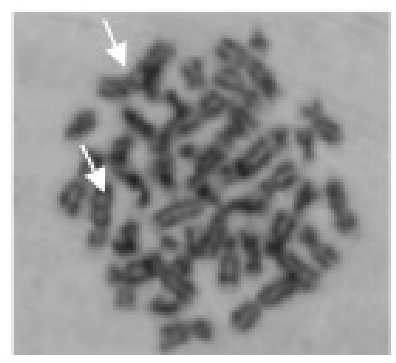

B)

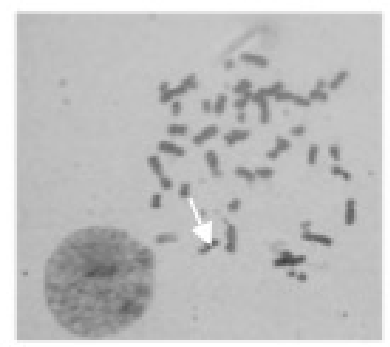

D)

Figure 2. Examples of the chromosomal aberrations found in workers exposed to the tanning process. (A) Chromosomes in ring (a) and acentric ring (ac) forms; (B) dicentric and tricentric chromosomes; (C) terminal and interstitial deletions; and (D) chromatid-type aberrations. 
In addition, the current study revealed that, as with CAs, the frequency of MN in binucleated cells was higher in exposed individuals. Increased prevalence of MN in tannery workers has already been documented in studies carried out in Portugal, Morocco, and India (Medeiros et al., 2003; Hilali et al., 2008; Balachandar et al., 2010). This finding confirms the clastogenicity evident from the CAs identified in this study.

A recent meta-analysis aiming to evaluate the role of $\mathrm{MN}$ as cancer biomarkers concluded that there exists a need to improve the design of studies relating to such research, since most investigations include several variables hindering the use of $\mathrm{MN}$ as reliable markers (Cardinale et al., 2012). However, according to larmarcovai et al. (2008), Bonassi et al. (2011), and KirschVolders et al. (2014), the presence of MN is an important measure of chromosomal damage and is linked to environmental factors and lifestyle habits.

Several products used in the processing of animal hides may be implicated in the chromosomal damage found in this study. However, chromium in particular stands out as one of those potentially responsible. Hexavalent chromium's ability to interact with human DNA and promote the formation of adducts has been well established (Salnikow and Zhitkovich, 2008). The formation of DNA adducts creates instability in the replication fork, leading to chromosomal breaks (Kirsch-Volders et al., 2014). Moreover, several studies in which chromium levels have been measured in tannery workers have found large amounts in biological samples (Randall and Gibson, 1989; Stupar et al., 1999; Medeiros et al., 2003; Ambreen et al., 2014).

Our investigation did not assess chromium concentration in the individuals studied, therefore we cannot connect the genotoxicity of this metal to the results obtained. However, using these data, we can infer that tannery workers sustain a greater amount of genetic damage than unexposed individuals. Contact with an environmental factor in the workplace may contribute to the development of these alterations. This hypothesis reinforces the suspicion surrounding chromium and establishes the need for more thorough biomonitoring studies. This will enable a better understanding of the relationship between environmental exposure and genetic anomalies, and encourage the adoption of preventive measures against future damage.

\section{REFERENCES}

Ambreen K, Khan FH, Bhadauria S and Kumar S (2014). Genotoxicity and oxidative stress in chromium-exposed tannery workers in North India. Toxicol. Ind. Health 30: 405-414.

Balachandar V, Arun M, Mohana Devi S, Velmurugan P, et al. (2010). Evaluation of the genetic alterations in direct and indirect exposures of hexavalent chromium [Cr(VI)] in leather tanning industry workers North Arcot District, South India. Int. Arch. Occup. Environ. Health 83: 791-801.

Bonassi S, Znaor A, Norppa H and Hagmar L (2004). Chromosomal aberrations and risk of cancer in humans: an epidemiologic perspective. Cytogenet. Genome Res. 104: 376-382.

Bonassi S, Norppa H, Ceppi M, Strömberg U, et al. (2008). Chromosomal aberration frequency in lymphocytes predicts the risk of cancer: results from a pooled cohort study of 22358 subjects in 11 countries. Carcinogenesis 29: 1178-1183.

Bonassi S, El-Zein R, Bolognesi C and Fenech M (2011). Micronuclei frequency in peripheral blood lymphocytes and cancer risk: evidence from human studies. Mutagenesis 26: 93-100.

Brazilian Institute of Geography and Statistics (IBGE) (2014). Informações sobre os municipios brasileiros. Available at [http:// www.cidades.ibge.gov.br/xtras/home.php]. Accessed March 4, 2015.

Cardinale F, Bruzzi P and Bolognesi C (2012). Role of micronucleus test in predicting breast cancer susceptibility: a systematic review and meta-analysis. Br. J. Cancer 106: 780-790.

Fenech M (2007). Cytokinesis-block micronucleus cytome assay. Nat. Protoc. 2: 1084-1104.

Hamamy HA, Al-Hakkak ZS and Hussain AF (1987). Chromosome aberrations in workers at a tannery in Iraq. Mutat. Res. 189: 395-398.

Hilali A, Anane R, Jaaouani N, Creppy EE, et al. (2008). Cytogenetic analysis of tannery workers in Morocco. J. Appl. Toxicol. 
28: 439-442.

larmarcovai G, Ceppi M, Botta A, Orsière T, et al. (2008). Micronuclei frequency in peripheral blood lymphocytes of cancer patients: a meta-analysis. Mutat. Res. 659: 274-283.

Kirsch-Volders M, Bonassi S, Knasmueller S, Holland N, et al. (2014). Commentary: critical questions, misconceptions and a road map for improving the use of the lymphocyte cytokinesis-block micronucleus assay for in vivo biomonitoring of human exposure to genotoxic chemicals-a HUMN project perspective. Mutat. Res. Rev. Mutat. Res. 759: 49-58.

Medeiros MG, Rodrigues AS, Batoréu MC, Laires A, et al. (2003). Elevated levels of DNA-protein crosslinks and micronuclei in peripheral lymphocytes of tannery workers exposed to trivalent chromium. Mutagenesis 18: 19-24.

Monteiro Neto MA, Lazaro CC, Tavares DC and Cecchi AO (2010). Frequency of chromosomal aberrations in peripheral lymphocytes of tannery workers in Brazil. Environ. Toxicol. Pharmacol. 29: 3-6.

Moorhead PS, Nowell PC, Mellman WJ, Battips DM, et al. (1960). Chromosome preparations of leukocytes cultured from human peripheral blood. Exp. Cell Res. 20: 613-616.

Norppa H, Bonassi S, Hansteen IL, Hagmar L, et al. (2006). Chromosomal aberrations and SCEs as biomarkers of cancer risk. Mutat. Res. 600: 37-45.

Pastor S, Creus A, Parrón T, Cebulska-Wasilewska A, et al. (2003). Biomonitoring of four European populations occupationally exposed to pesticides: use of micronuclei as biomarkers. Mutagenesis 18: 249-258.

Preston RJ, San Sebastian JR and McFee AF (1987). The in vitro human lymphocyte assay for assessing the clastogenicity of chemical agents. Mutat. Res. 189: 175-183.

Randall JA and Gibson RS (1989). Hair chromium as an index of chromium exposure of tannery workers. Br. J. Ind. Med. 46: 171-175.

Rastogi SK, Pandey A and Tripathi S (2008). Occupational health risks among the workers employed in leather tanneries at Kanpur. Indian J. Occup. Environ. Med. 12: 132-135.

Salnikow K and Zhitkovich A (2008). Genetic and epigenetic mechanisms in metal carcinogenesis and cocarcinogenesis: nickel, arsenic, and chromium. Chem. Res. Toxicol. 21: 28-44.

Savage JR (1976). Classification and relationships of induced chromosomal structural changes. J. Med. Genet. 13: $103-122$.

Shahzad K, Akhtar S and Mahmud S (2006). Prevalence and determinants of asthma in adult male leather tannery workers in Karachi, Pakistan: a cross sectional study. BMC Public Health 6: 292.

Stupar J, Vrtovec M, Kocijancic A and Gantar A (1999). Chromium status of tannery workers in relation to metabolic disorders. J. Appl. Toxicol. 19: 437-446.

Tanning Industry Center of Brazil (CIC) (2013). Distribuição por estado da exportação de couros e peles. Available at http:// www.cicb.org.br/wp-content/uploads/2013/03/Estados-Exportadores.jpg. Accessed March 2, 2015.

Veyalkin I and Gerein V (2006). Retrospective cohort study of cancer mortality at the Minsk Leather Tannery. Ind. Health 44: 69-74. 